\title{
TRIM28 activates autophagy and promotes cell proliferation in glioblastoma
}

This article was published in the following Dove Medical Press journal: OncoTargets and Therapy

\author{
Yong Peng \\ Mingming Zhang \\ Zhongzhong Jiang \\ Yugang Jiang
}

Department of Neurosurgery, The Second Xiangya Hospital, Central South University,

Changsha, Hunan, China
Correspondence: Yugang Jiang

Department of Neurosurgery,

The Second Xiangya Hospital, Central

South University, 139 People's Middle

Road, Changsha, Hunan 4100II, China

Tel +8673185295110

Fax +86 73185295810

Email foxpy195@csu.edu.cn
Background: Tripartite motif-containing protein 28 (TRIM28) is a transcriptional corepressor involved in the regulation of several cancers, including glioma. It has been reported that TRIM28 takes part in the process of autophagy. However, its effect on the autophagy and cell proliferation in gliomas has not been elucidated. Here, we report a novel tumor cell proliferation mechanism in which TRIM28-regulated autophagy promotes glioma tumor cell proliferation. Materials and methods: We analyzed the expressions of TRIM28 and LC3 in different WHO grades of gliomas by IHC assays. We then knocked down and overexpressed TRIM28 or knocked down ATG5 in U251 cells and confirmed its roles in autophagy and cell proliferation via cell counting, immunofluorescence, and Western blot.

Results: The results showed that TRIM28 and autophagy levels were significantly increased with the progression of tumor grade in glioma. TRIM28 promoted glioblastoma cell proliferation. Knockdown of TRIM28 inhibited autophagy in glioblastoma cells. Meanwhile, TRIM28 promoted glioblastoma cell proliferation by modulating TRIM28.

Conclusion: These data demonstrated that TRIM28 activates autophagy and increases cell proliferation in glioma.

Keywords: TRIM28, autophagy, glioblastoma, proliferation

\section{Introduction}

Gliomas are the most common intracranial tumors, accounting for more than half of such tumors, with an incidence of 0.59-3.69 per 100,000 people after age adjustment. ${ }^{1}$ They are characterized by high malignancy, poor prognosis, and high mortality. ${ }^{2}$ With an increasing malignancy grade of gliomas, their multiplication ability becomes enlarged. ${ }^{3}$ Despite the multitude of mechanisms that have been proposed to explain the proliferation of tumor, the exact mechanism, however, remains to be explained.

Autophagy is a highly conserved process of intracellular component degradation, providing cells with opportunities to overcome starvation, hypoxia, and metabolic stress, which is essential for maintaining homeostasis in normal cells. ${ }^{4}$ Although autophagy has been reported to be a protective process in normal cells, this process is a double-edged sword in tumor cells. On the one hand, activation of autophagy could promote autophagic programmed cell death and kill tumor cells, and on the other hand, tumor cells are overconsumptive and tend to develop autophagic process to adapt to the relatively infertile tumor microenvironment. ${ }^{5}$ Several lines of evidence have demonstrated that the induction of autophagy might promote the proliferation of tumor cells. ${ }^{6}$ However, the relationship and mechanism of autophagy and cell proliferation are not yet completely clear.

Tripartite motif-containing (TRIM) superfamily members are involved in a broad range of biological processes, including carcinogenesis regulation. ${ }^{7}$ For example, 
TRIM19 and TRIM59 were shown to participate in liver and gastric cancers, ${ }^{8,9}$ respectively. TRIM protein 28 (TRIM28) is a transcriptional corepressor involved in the regulation of cancer as well. A recent report demonstrated that TRIM28 could regulate protein posttranslational modification and take part in the process of autophagy. ${ }^{10}$ However, its effects on autophagy and cell proliferation in gliomas have not been elucidated. Therefore, it is highly desirable to study TRIM28mediated autophagy in the cell proliferation of glioma, which might provide new insights into the treatment of glioma.

In the present study, we report a novel tumor cell proliferation mechanism in which TRIM28-regulated autophagy promotes glioma tumor cell proliferation. We first investigated TRIM28 and LC3 levels in human glioma tissues, and found significant TRIM28 expression and autophagy activation in glioma tissues, which correlated with the grade of human glioma. Exogenous TRIM28 increased the proliferation of glioblastoma cells, whereas knocking down endogenous TRIM28 inhibited cell proliferation. More importantly, the application of TRIM28 knockdown antagonized autophagy. To understand the mechanisms of the tumor cell proliferation induced by TRIM28 and autophagy, we knocked down endogenous ATG5 and found that this mitigated TRIM28-regulated cell proliferation. Finally, we provided preliminary evidence that the TRIM28-autophagy pathway plays a central role in the tumor cell proliferation. Our results suggest potential uses for anti-TRIM28 and anti-autophagy therapeutic strategies as adjuvant therapies for glioma patients, however, further basic and clinical investigations are needed.

\section{Materials and methods Glioma tissue samples}

A total of 65 human glioma tissue samples were obtained from the Department of Neurosurgery of the Second Xiangya Hospital of Central South University from October 2013 to June 2017, including 23 grade II tumors, 16 grade III tumors, and 26 grade IV tumors. The glioma specimens were verified and classified according to the WHO classification standard and were verified by two clinical pathologists. Our study was conducted in accordance with the Declaration of Helsinki and approved by the Medical Ethics Committee of the Second Xiangya Hospital of Central South University. Written informed consent was obtained from all patients.

\section{Immunohistochemical staining}

Immunohistochemical staining was performed as previously described. ${ }^{11}$ Human glioma tissue samples were fixed with $4 \%$ formaldehyde overnight, and then paraffin-embedded using Leica EG1150. Paraffin-embedded glioma tissues were sectioned to $5-\mu \mathrm{m}$ thickness, and immunohistochemistry was performed according to the standard procedure. Sections were incubated with primary antibody against TRIM28 (dilution 1:500, ab109545; Abcam, Cambridge, UK) and LC3 (dilution 1:500, ab192890; Abcam) at $4^{\circ} \mathrm{C}$ overnight. After rinsing with PBS, sections were incubated with horseradish peroxidase-conjugated goat anti-rabbit secondary antibodies (dilution 1:1,000, ab6721; Abcam), then reacted with DAB (Sigma-Aldrich Co., St Louis, MO, USA). Immunohistochemical staining score was processed as follows: a staining intensity value of $0=$ negative, $1=$ weak, $2=$ moderate, and $3=$ strong; percentage of positive cells value of $0=$ negative, $1=10 \%$ positive cells, $2=11 \%-50 \%$ positive cells, $3=51 \%-80 \%$ positive cells, and $4=$ more than $80 \%$ positive cells. Five random visual fields of each glioma section were used for the score evaluation.

\section{Cell culture}

The human glioblastoma cell line U251 was purchased from the Shanghai Cell Bank of the Chinese Academy of Sciences (Shanghai, China), and were routinely cultured in DMEM (Thermo Fisher Scientific, Waltham, MA, USA) with 10\% FBS (Thermo Fisher Scientific) and maintained at $37^{\circ} \mathrm{C}$ with $5 \% \mathrm{CO}_{2}$. Starvation treatment was applied with Hank's balanced salt solution (Thermo Fisher Scientific) for 6 hours.

\section{siRNA and vector transfection}

TRIM28, ATG5, and control siRNAs were synthesized by RiboBio Co., Ltd. (Guangzhou, China). The sequences of siRNAs were as follows: TRIM28-1 (5'-CCAAAGACAUCG UGGAGAAUUAUUU-3'), TRIM28-2 (5'-CAGUGCUG CACUAGCUGUGAGGAUA-3'), ATG5 (5'-UCGAGAUGU GUGGUUUGACGGAAUU-3'), and control siRNA (5'-UAAGGCUAUGAAGAGAUAC-3'). TRIM28 overexpression vector (TRIM28-myc) was provided by Dr Tan Jieqiong. The siRNAs or vectors were transfected into U251 cells for 48 hours using Lipofectamine 2000 (Thermo Fisher Scientific) according to the manufacturer's instructions.

\section{Cell proliferation assay}

Cell proliferation was determined by cell counting. U251 cells were seeded into 6-well plates at $10^{5}$ cells/well, and were incubated overnight. At the desired time-points after treatment, the cells were digested by trypsin into single cell suspensions, and Trypan blue was added (final concentration $0.04 \%$ ). Cells were counted by a hemocytometer under microscopy. 


\section{Immunofluorescence staining}

Immunofluorescence staining was performed as previously described. ${ }^{12}$ U251 cells were seeded into glass slides in 24-well culture plates at $10^{5}$ cells/well for 24 hours and subsequently treated with desired reagents. At the desired time-points after treatment, the cells were fixed with $4 \%$ formaldehyde, permeabilized with $0.5 \%$ Triton X-100, then stained with the primary antibodies against LC3 (dilution 1:1,000, ERP18709; Abcam) overnight, and finally labeled with anti-rabbit IgG conjugated with Alexa Fluor 488 (A-11034; Thermo Fisher Scientific). The cells were observed under confocal microscopy (Leica TCS SP8; Leica Microsystems, Wetzlar, Germany), and images were obtained. Five random visual fields of each slides were used for the evaluation.

\section{Protein preparation and Western blot analysis}

Proteins were extracted from U251 cells with RIPA cell lysis buffer. Extracts were centrifuged at $12,000 \times \mathrm{g}$ for 15 minutes at $4{ }^{\circ} \mathrm{C}$, and the supernatant proteins were collected. Western blot analysis was performed as previously described. ${ }^{13}$ The protein concentrations were measured using a bicinchoninic acid protein assay kit (Dojindo Laboratories, Kumamoto, Japan). Total protein (10-20 $\mu$ g per lane) was separated on $15 \%$ SDS-PAGE and transferred to a polyvinylidene fluoride membrane (EMD Millipore, Billerica, MA, USA). The PVDF membrane was blocked in 5\% skimmed milk in Tris-buffered saline Tween ([TBST], $\mathrm{pH}$ 7.6), and then incubated overnight at $4{ }^{\circ} \mathrm{C}$ with primary antibodies against LC3 (dilution 1:1,000, ab192890; Abcam), TRIM28 (dilution 1:1,000, ab109545; Abcam), ATG5 (dilution 1:1,000, ab109490; Abcam), or beta-actin (dilution 1:2,000, ab49900; Abcam). Subsequently, the membrane was rinsed thrice with TBST and incubated for 1 hour at room temperature with horseradish peroxidase-conjugated goat anti-rabbit or anti-mouse secondary antibodies (dilution 1:2,000, ab6721 and ab6728; Abcam). After rinsing thrice with TBST, the membrane was incubated with enhanced chemiluminescence substrate reagent (Thermo Fisher Scientific; Pierce) and pictures were recorded using Healthcare ImageQuant LAS 500 (GE Healthcare Bio-Sciences Corp., Piscataway, NJ, USA). These experiments were repeated at least three times.

\section{Statistical analysis}

All data are expressed as the means \pm SD. Statistical analysis was performed using SPSS 19.0 (IBM Corporation, Armonk, NY, USA) and GraphPad Prism 5 (GraphPad Software Inc., La Jolla, CA, USA). The differences among the groups were evaluated with one-way ANOVA followed by the Tukey post hoc test. $P<0.05$ was considered statistically significant.

\section{Results \\ TRIM28 and autophagy levels were significantly increased with the progression of tumor grade in glioma}

To investigate the role of TRIM 28 and autophagy in the progress of glioma, we examined TRIM28 and autophagy marker LC3 levels in human glioma specimens by immunohistochemical staining. As shown in Figure 1A and B, TRIM28 expression was remarkably higher in WHO Grade III and IV gliomas than in Grade II gliomas. Meanwhile, we also confirmed that autophagy was activated in high grade gliomas. The expressions of TRIM 28 and LC 3 positively correlated with the WHO grade of the glioma (Figure 1C). Importantly, Spearman's rho rank correlation test showed that TRIM28 and LC3 were positively correlated as well. All the correlation indexes were $>0.7$, indicating a strong positive relationship. These data demonstrated that TRIM28 was significantly increased, and was associated with autophagy activation in glioma.

\section{TRIM28 levels correlated with the proliferation of glioblastoma cells}

It is generally accepted that high-grade glioma is characterized by high proliferation. To verify whether TRIM 28 affected the proliferation of glioma, we performed TRIM 28 gene knockdown or overexpression, and then observed the proliferation of glioblastoma cells. As shown in Figure 2A and B, after transfection with TRIM28 siRNA, we detected a significant decrease in TRIM28 expression by Western blotting, and meanwhile, the proliferation of glioblastoma cells were inhibited. By contrast, TRIM28-myc plasmid transient transfection promoted the proliferation of glioblastoma cells (Figure 2C and D). These results suggested that TRIM28 may be involved in regulating the cell proliferation of glioblastoma cells.

\section{TRIM28-regulated autophagy in glioblastoma cells}

We observed the relationship between TRIM28 and LC3; however, the specific regulatory mechanism between them is unknown. To explore the mechanisms of TRIM28 in regulating proliferation, we investigated whether TRIM28 regulates cell proliferation-related autophagy signal using a green-LC3 puncta-formation assay and an LC3 conversion assay. Using immunofluorescence, green-LC3 localization was examined, and the conversion of LC3-I into LC3-II was detected by 
A

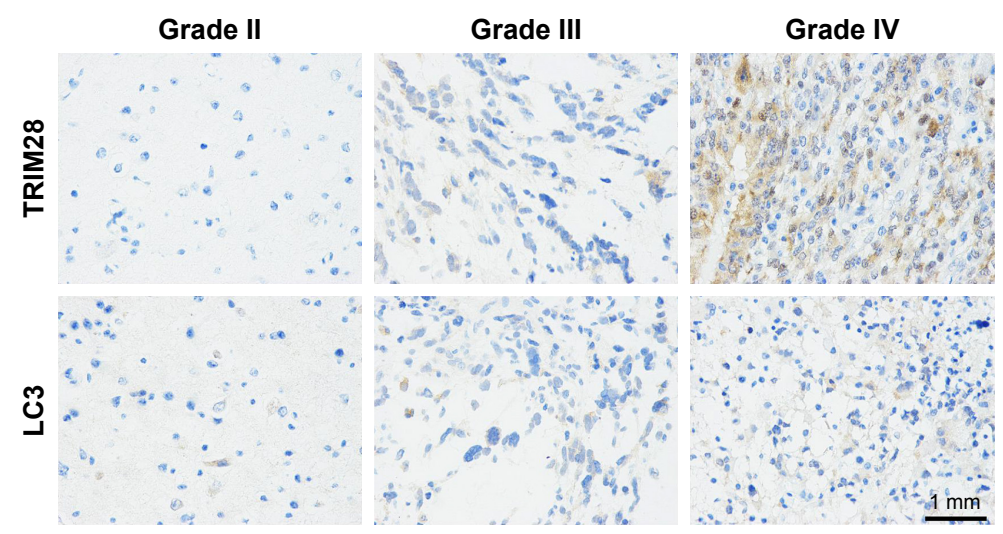

B
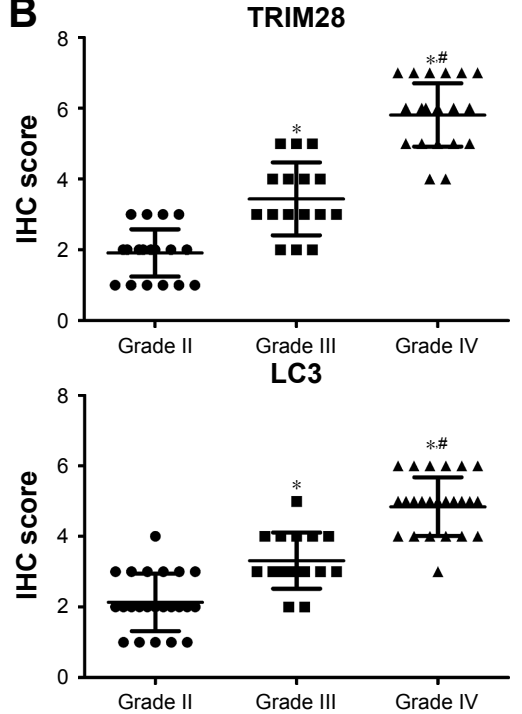

C

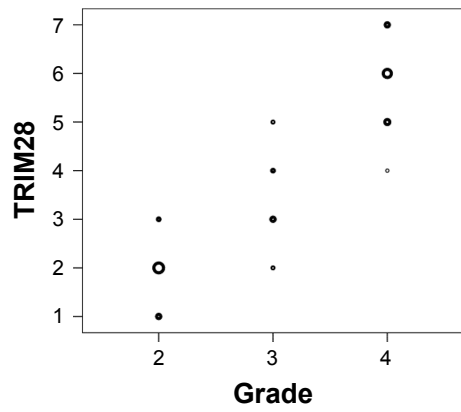

0.857

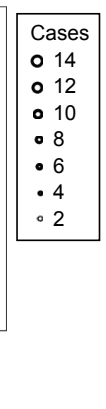

\begin{tabular}{ll|}
\hline Cases \\
0 & 14 \\
0 & 12 \\
0 & 10 \\
0 & 8 \\
0 & 6 \\
$:$ & 4 \\
0 & 2 \\
\hline
\end{tabular}

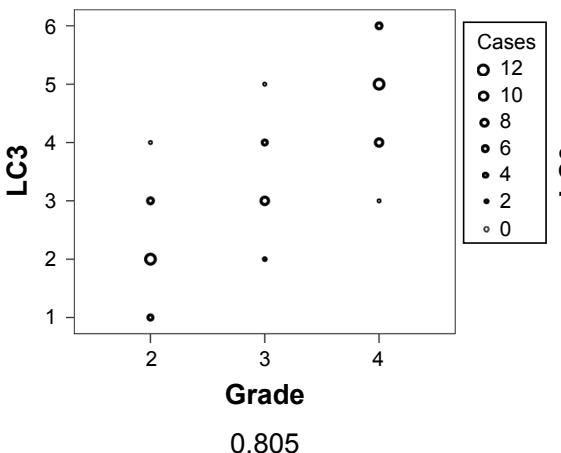

0.805

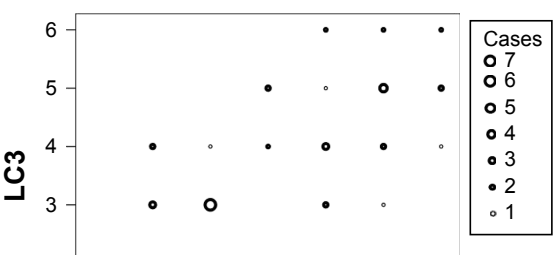

Spearman's correlation test

Figure I Comparison of TRIM28 and autophagy marker LC3 expression levels in glioma tissues with different WHO grades.

Notes: A total of 65 human glioma tissue samples were obtained, and the TRIM28 and LC3 expression levels were analyzed by IHC. (A) The TRIM28 and LC3 expression levels in different WHO grades of glioma. (B) Quantification of IHC staining of glioma tissues with different $W H O$ grades $\left(P<0.05 ; * P<0.05\right.$ vs grade II group; ${ }^{*}<0.05$ vs grade III group). (C) Spearman's rho rank correlation tests of TRIM28, LC3, and grades ( 0.857 between TRIM28 and WHO grades, 0.805 between LC3 and WHO grades, and 0.706 between LC3 and TRIM28).

Abbreviation: TRIM28, tripartite motif-containing protein 28.

Western blotting. As shown in Figure 3A, there was a significant increase in green-LC3 puncta in cells treated with starvation, which was reduced by TRIM 28 gene knockdown; this result was confirmed by the quantification of green-LC3 puncta per cell. Consistent with the immunofluorescence assay, starvation led to a significant upregulation of LC3-II and TRIM 28 gene knockdown blocked the starvation-induced increase in LC3-II (Figure 3B). Furthermore, TRIM28 overexpression increased the number of green-LC3 puncta and the $\mathrm{LC} 3$ conversion ratio (Figure $3 \mathrm{C}$ and $\mathrm{D}$ ). These results suggested that TRIM28 regulates autophagy activation in glioblastoma cells.

\section{TRIM28 regulated proliferation through autophagy in glioblastoma cells}

We next assayed whether TRIM28 regulates glioblastoma cell proliferation by autophagy. We used ATG5-siRNA to inhibit autophagy. We found that autophagy inhibition abrogated TRIM28 upregulation-induced cell proliferation (Figure 4). These data confirmed that TRIM28-regulated proliferation depended on autophagy activation in glioblastoma cells.

\section{Discussion}

TRIM28 is a member of the TRIM family, which has been shown to play pleiotropic roles in tumor proliferation. ${ }^{14}$ TRIM28 acts as an oncogene in gliomas; ${ }^{15}$ however, the molecular mechanisms remain unclear. Autophagy promotes glioma cell proliferation during tumor progression. ${ }^{16}$ Recently obtained evidence has demonstrated that TRIM 28 regulates the process of autophagy. ${ }^{17}$ In the present study, we found that significant TRIM28 expression and autophagy activation in glioma tissues correlated with the grade of glioma. Meanwhile, TRIM28 promoted cell proliferation and activated 
A

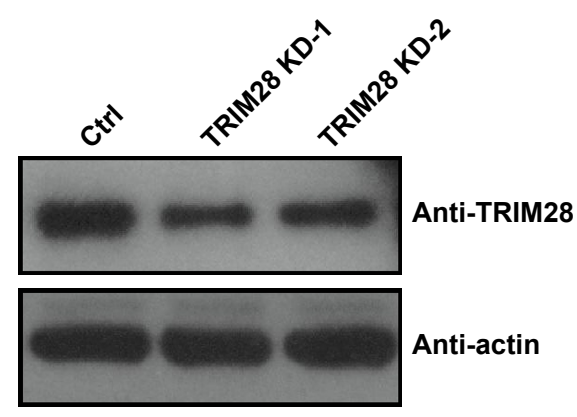

C

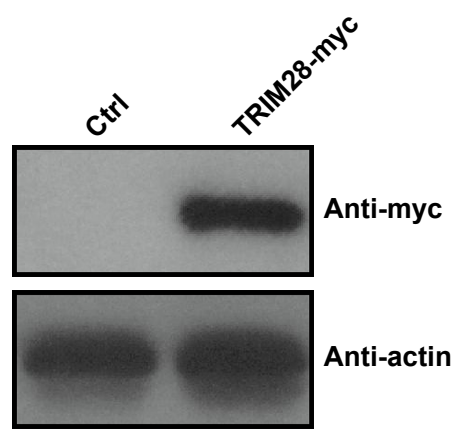

B 10
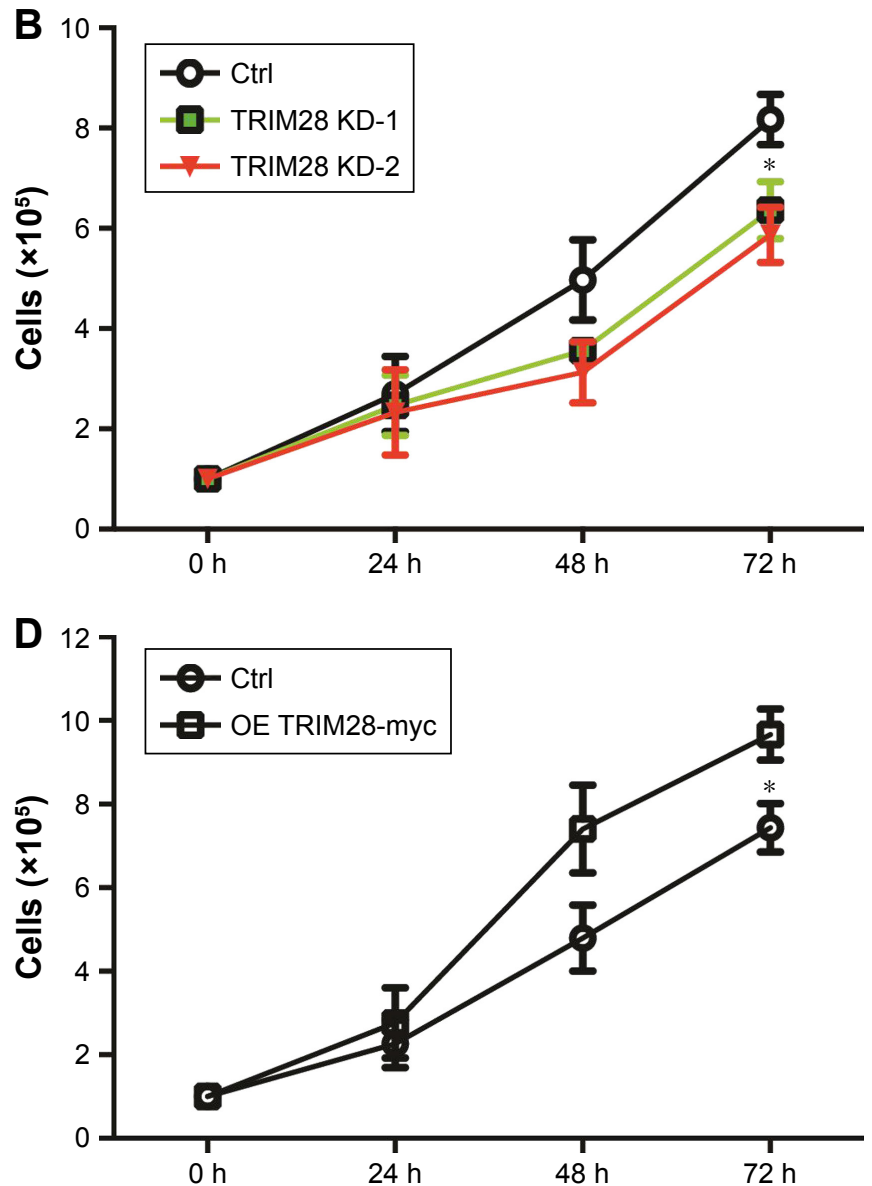

Figure 2 TRIM28 affects glioblastoma cell proliferation.

Notes: TRIM28 siRNA or TRIM28-myc was transfected into U25I cells, and control siRNA and pCMV-myc transfected cells were used as the controls. (A and C) Western blot analysis of TRIM28 expression in TRIM28-myc- or TRIM28 siRNA-infected cells. (B and D) cell proliferation measured using cell counting after TRIM28-myc or TRIM28 siRNA transfection in $U 25$ I cells. ( $P<0.05 ; * P<0.05$ vs control group).

Abbreviations: Ctrl, control; TRIM28, tripartite motif-containing protein 28.

autophagy. More importantly, exogenous ATG5 knockdown mitigated TRIM28-induced cell proliferation. Thus, our findings may provide a novel tumor cell proliferation mechanism by targeting TRIM28-regulated autophagy in glioma.

TRIM28, previously known as Krüppel-associated boxassociated protein 1, is a nuclear corepressor for kruppelassociated box domain-containing zinc finger proteins. ${ }^{18}$ TRIM28 participates in many aspects of cellular biology, either promoting proliferation or leading to anti-proliferation. Immunohistochemical analysis revealed that TRIM28 levels were significantly higher in lung, liver, gastric, breast, and prostate cancers. ${ }^{19-21}$ In some cancers, TRIM28 acts as a tumor promoting gene. Several reports showed that TRIM28 expression was positively correlated with cancer prognosis, such as in ovarian and lung cancers. ${ }^{22,23}$ However, TRIM28 acts as a tumor suppressor in some cancers as well. Herquel et al reported that inactivation of hepatocyte TRIM28 could promote hepatocellular tumorigenesis. ${ }^{24}$ Their report suggested that TRIM28 indeed affects cell proliferation. To confirm the correlation of TRIM28 and cell proliferation in glioma, we observed TRIM28 expression in different grades of glioma, and overexpressed or knocked down TRIM28 in U251 cells. Those results indicated that TRIM28 promoted cell proliferation and was related to glioma progression. Qi et al reported that TRIM28 expression was significantly higher in glioma, and deletion of TRIM28 has been recently reported to suppress tumor growth. ${ }^{25}$

Autophagy is a stress-induced process that degrades bulk cytoplasmic contents, abnormal protein aggregates, and excess or damaged organelles. ${ }^{26} \mathrm{LC} 3$ is a soluble protein and is distributed ubiquitously in mammalian tissues and cultured cells. During autophagy, LC3-I (cytosolic form) is conjugated to phosphatidylethanolamine and forms LC3-II (LC3-phosphatidylethanolamine conjugate), which can be recruited to autophagosomal membranes. Detection of LC3 by immunofluorescence or Western blot has become a reliable method for monitoring autophagy. ${ }^{27}$ Recently, TRIM28 was reported to regulate autophagy as well. However, whether the effect 
A
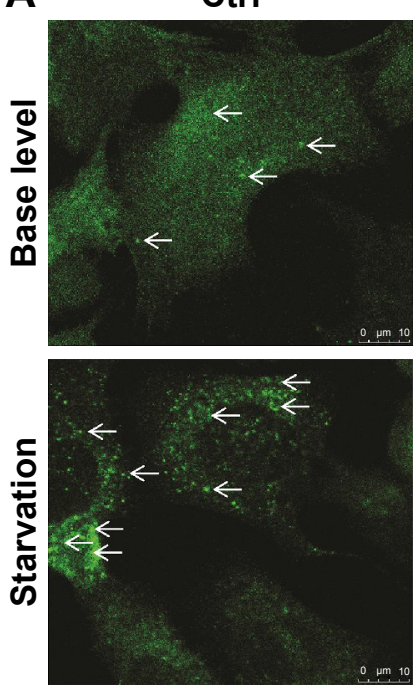

C

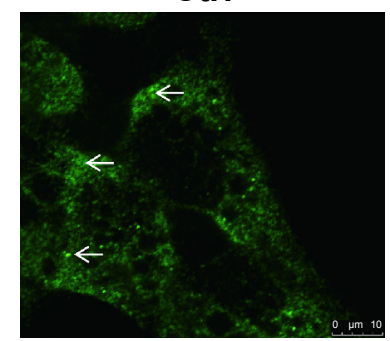

TRIM28 KD
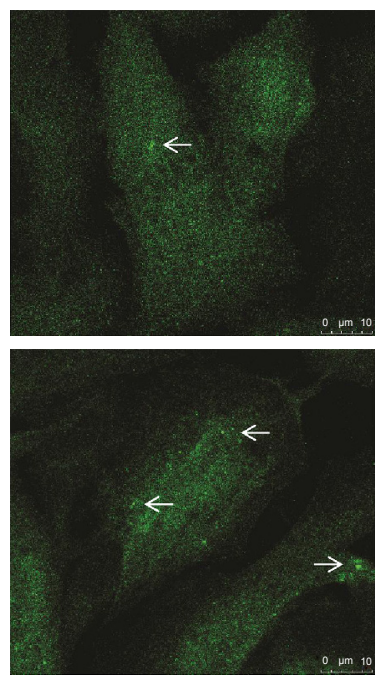

TRIM28-myc

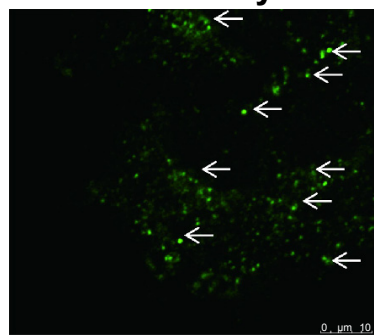

B

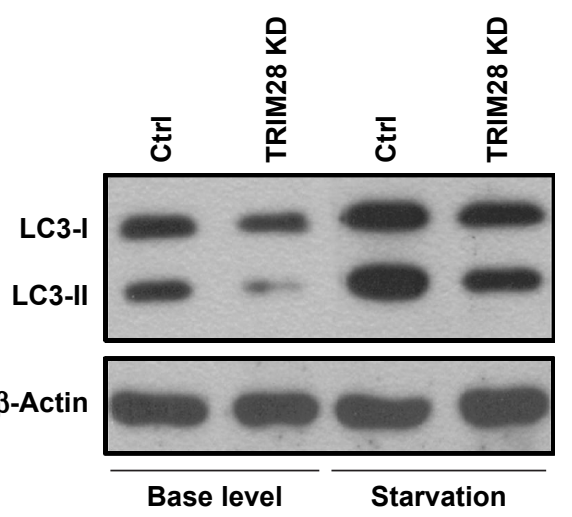

D

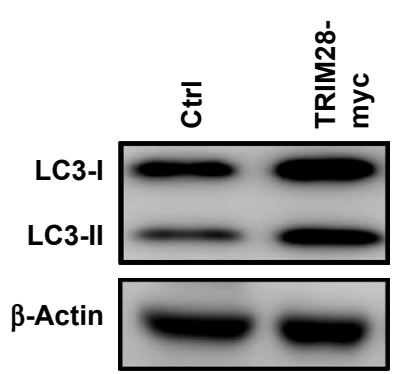

Figure 3 TRIM28 regulates autophagy in U25 I cells.

Notes: (A and C) TRIM28 siRNA or TRIM28-myc transfection regulated autophagy in U25I cells. Green staining LC3 puncta formation were labeled with arrows, indicating autophagy activation. (B and D) Western blot analysis of LC3-I to LC3-Il conversion after TRIM28 siRNA or TRIM28-myc transfection in U25I cells.

Abbreviations: Ctrl, control; TRIM28, tripartite motif-containing protein 28.

of TRIM28 in cell proliferation is mediated by autophagy remains unknown. We hypothesized that TRIM28 is a potent autophagy inducer in glioma and regulates glioblastoma cell proliferation. To prove this hypothesis, we first confirmed that knockdown of TRIM28 inhibited autophagy activation. Further, inhibition of endogenous ATG5 clarified the role of autophagy in TRIM28-mediated cell proliferation. Cancer cells can recycle intracellular proteins and organelles during
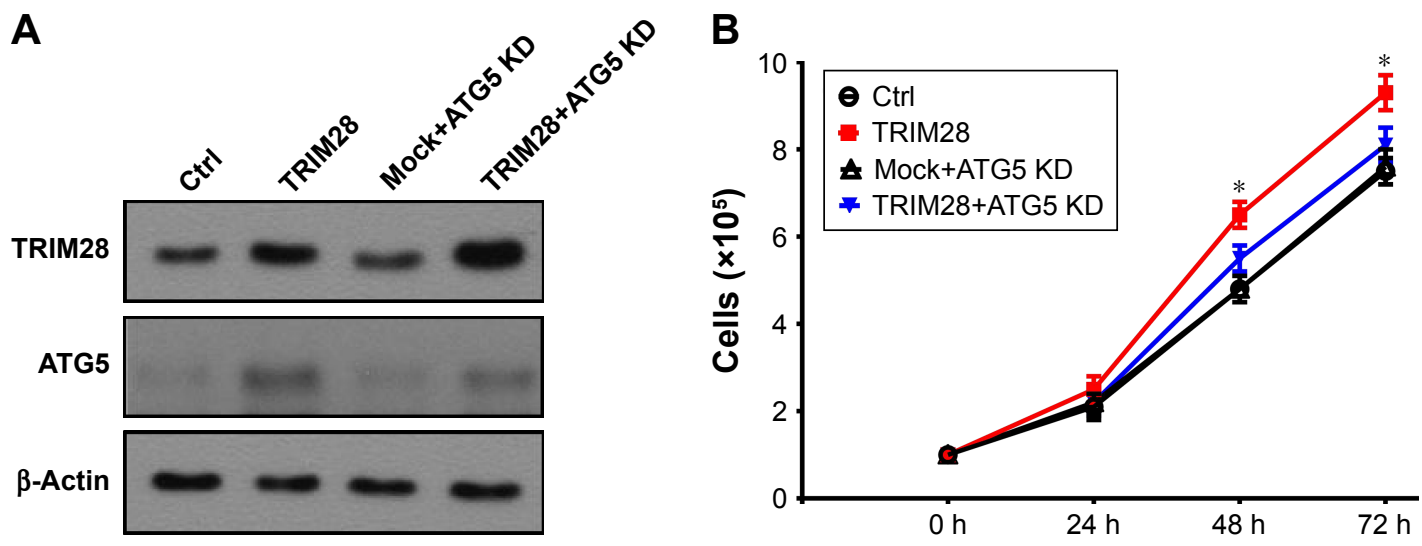

Figure 4 TRIM28 affects glioblastoma cell proliferation by modulating ATG5.

Notes: TRIM28-myc and/or ATG5 siRNA was transfected into U25I cells. (A) Western blot analysis of TRIM28 and protein expression in TRIM28-myc and/or ATG siRNA infected cells. (B) Measuring cell proliferation using cell counting after TRIM28-myc and/or ATG siRNA transfection in U25I cells. $(P<0.05$; $* P<0.05$ vs TRIM28+ATG5 KD group).

Abbreviations: Ctrl, control; TRIM28, tripartite motif-containing protein 28. 
autophagy to provide energy for cell survival. ${ }^{28}$ TRIM28 regulated autophagy at several distinct levels. On the one hand, TRIM28 protein was reported to play a significant role in coordination of autophagosome formation, ${ }^{29}$ where TRIM28 was also involved in selective degradation of mitochondria by autophagy. ${ }^{30}$ On the other hand, the effect of TRIM 28 on autophagy can be converted by MAGE-A3/6 through targeting AMPK for degradation. ${ }^{17}$ Certainly, there were some limitations to our current study. We only used U251 cells as a cell model to verify the role of TRIM28 in autophagy and cell proliferation, and using another glioblastoma cell line may better substantiate the findings. Moreover, it is not known whether the mechanism by which TRIM28 regulates autophagy is similar in glioma cells to that of other models; our plan for a future study is to investigate this mechanism and to further explore how TRIM28 exerts its role of promoting autophagy and inhibiting cell proliferation in glioma.

\section{Conclusion}

In summary, our study demonstrated that TRIM28 was strongly associated with the grade of glioma in patients. TRIM28 promoted cell proliferation and activated autophagy. In addition, we showed that TRIM28 contributed to cell proliferation via autophagy. Altogether, our data suggest that anti-TRIM28 and anti-autophagy therapeutic strategies might be of potential use as adjuvant therapies for glioma patients; however, further basic and clinical investigations are needed.

\section{Disclosure}

The authors report no conflicts of interest in this work.

\section{References}

1. Ostrom QT, Gittleman H, Stetson L. Epidemiology of gliomas. Cancer Treat Res. 2015;163:1-14.

2. Omuro A, Deangelis LM. Glioblastoma and other malignant gliomas: a clinical review. JAMA. 2013;310(17):1842-1850.

3. Davis ME, Stoiber AM, Stoiber AM. Glioblastoma multiforme: enhancing survival and quality of life. Clin J Oncol Nurs. 2011;15(3):291-297.

4. Hurley JH, Young LN. Mechanisms of autophagy initiation. Annu Rev Biochem. 2017:86225-86244.

5. Guo JY, Xia B, White E. Autophagy-mediated tumor promotion. Cell. 2013;155(6):1216-1219.

6. Aredia F, Guamán Ortiz LM, Giansanti V, Scovassi AI, et al. Autophagy and cancer. Cells. 2012;1(3):520-534.

7. Hatakeyama S. TRIM Family proteins: roles in autophagy, immunity, and carcinogenesis. Trends in Biochem Sci. 2017;42(4): 297-311.

8. Chung YL, Wu ML, Ml W. Promyelocytic leukaemia protein links DNA damage response and repair to hepatitis B virus-related hepatocarcinogenesis. J Pathol. 2013;230(4):377-387.

9. Zhou Z, Ji Z, Wang Y, et al. TRIM59 Is Up-regulated in gastric tumors, promoting ubiquitination and degradation of $\mathrm{p} 53$. Gastroenterology. 2014;147(5):1043-1054.
10. Yang Y, Fiskus W, Yong B, et al. Acetylated hsp70 and KAP1mediated Vps34 SUMOylation is required for autophagosome creation in autophagy. Proc Natl Acad Sci U S A. 2013;110(17): 6841-6846.

11. Xue H, Yuan G, Guo X, et al. A novel tumor-promoting mechanism of IL6 and the therapeutic efficacy of tocilizumab: Hypoxia-induced IL6 is a potent autophagy initiator in glioblastoma via the p-STAT3MIR155-3p-CREBRF pathway. Autophagy. 2016;12(7):1129-1152.

12. Jiang Y, Gao M, Wang W, et al. Sinomenine hydrochloride protects against polymicrobial sepsis via autophagy. Int J Mol Sci. 2015;16(2):2559-2573.

13. Peng Y, Gao M, Jiang Y, et al. Angiogenesis inhibitor endostatin protects mice with sepsis from multiple organ dysfunction syndrome. Shock. 2015;44(4):357-364

14. Dalgaard K, Landgraf K, Heyne S, et al. Trim28 haploinsufficiency triggers Bi-stable epigenetic obesity. Cell. 2016;164(3):353-364.

15. Qi Z, Cai S, Cai J, et al. miR-491 regulates glioma cells proliferation by targeting TRIM28 in vitro. BMC Neurol. 2016;16(1):248.

16. Hombach-Klonisch S, Mehrpour M, Shojaei S. Glioblastoma and chemoresistance to alkylating agents: involvement of apoptosis, autophagy, and unfolded protein response. Pharmacol Ther. 2018: 18413-18441.

17. Pineda CT, Potts PR. Oncogenic MAGEA-TRIM28 ubiquitin ligase downregulates autophagy by ubiquitinating and degrading AMPK in cancer. Autophagy. 2015;11(5):844-846.

18. Sampath Kumar A, Seah MKY, Ling KY, et al. Loss of maternal Trim 28 causes male-predominant early embryonic lethality. Genes Dev. 2017;31(1):12-17.

19. Wang Y, Jiang J, Li Q, et al. KAP1 is overexpressed in hepatocellular carcinoma and its clinical significance. Int J Clin Oncol. 2016;21(5):927-933.

20. Czerwińska P, Shah PK, Tomczak K, et al. TRIM28 multi-domain protein regulates cancer stem cell population in breast tumor development. Oncotarget. 2017;8(1):863-882.

21. Wei C, Cheng J, Zhou B, et al. Tripartite motif containing 28 (TRIM28) promotes breast cancer metastasis by stabilizing TWIST1 protein. $S c i$ Rep. 2016;6(1):29822.

22. Cui Y, Yang S, Fu X, et al. High levels of KAP1 expression are associated with aggressive clinical features in ovarian cancer. Int J Mol Sci. 2014;16(1):363-377.

23. Liu L, Zhao E, Li C, et al. TRIM28, a new molecular marker predicting metastasis and survival in early-stage non-small cell lung cancer. Cancer Epidemiol. 2013;37(1):71-78.

24. Herquel B, Ouararhni K, Khetchoumian K, et al. Transcription cofactors TRIM24, TRIM28, and TRIM33 associate to form regulatory complexes that suppress murine hepatocellular carcinoma. Proc Natl Acad Sci USA. 2011;108(20):8212-8217.

25. Qi ZX, Cai JJ, Chen LC, et al. TRIM28 as an independent prognostic marker plays critical roles in glioma progression. J Neurooncol. 2016; 126(1):19-26.

26. Dikic I, Elazar Z. Mechanism and medical implications of mammalian autophagy. Nat Rev Mol Cell Biol. 2018;19(6):349-364.

27. Tanida I, Ueno T, Kominami E. LC3 and Autophagy. Methods Mol Biol. 2008:445:77-88.

28. Levy JMM, Towers CG, Thorburn A. Targeting autophagy in cancer. Nat Rev Cancer. 2017;17(9):528-542.

29. Yang Y, Fiskus W, Yong B, et al. Acetylated hsp70 and KAP1-mediated Vps34 SUMOylation is required for autophagosome creation in autophagy. Proc Natl Acad Sci U S A. 2013;110(17):6841-6846.

30. Barde I, Rauwel B, Marin-Florez RM, et al. A KRAB/KAP1-miRNA cascade regulates erythropoiesis through stage-specific control of mitophagy. Science. 2013;340(6130):350-353. 


\section{Publish your work in this journal}

OncoTargets and Therapy is an international, peer-reviewed, open access journal focusing on the pathological basis of all cancers, potential targets for therapy and treatment protocols employed to improve the management of cancer patients. The journal also focuses on the impact of management programs and new therapeutic agents and protocols on

patient perspectives such as quality of life, adherence and satisfaction. The manuscript management system is completely online and includes a very quick and fair peer-review system, which is all easy to use. Visit http://www.dovepress.com/testimonials.php to read real quotes from published authors.

Submit your manuscript here: http://www.dovepress.com/oncotargets-and-therapy-journal 